\title{
Nagelosteosynthese als Revisionseingriff nach Versagen der Plattenosteosynthese bei Unterarmschaftfrakturen
}

$\square$ Alexander Hofmann, Pol Maria Rommens

\section{Einleitung}

Diaphysäre Unterarmfrakturen erfordern in jedem Lebensalter eine wohlüberlegte und adäquate Behandlung, um der Gefahr eines Osteosyntheseversagens und verzögerter Bruchheilung vorbeugen zu können. Die Operationstechnik muss dabei den grundlegenden Prinzipien der Versorgung diaphysärer Frakturen gerecht werden und eine hohe biomechanische Angulations- und Rotationsstabilität gewährleisten. Der folgende Fall schildert eindrücklich die Problematik der LC-DC-Plattenosteosynthese bei instabilen Unterarmfrakturen.

\section{Klinischer Fall}

Der 31-jährige männliche Patient erlitt im Rahmen eines Sturzes beim Fußballspiel eine instabile Fraktur des linken Unterarmschafts (Abb.1). Zunächst erfolgte die operative Versorgung des Radius und der Ulna mittels zweier 6-Loch-LC-DC-Platten (Abb. 2). Bereits 2 Monate postoperativ zeigen sich eine zunehmende Achsenfehlstellung der Ulna sowie deutliche Zeichen einer hypertrophen Kallusbildung im Bereich beider Frakturen (Abb. 3). Ein Jahr postoperativ ist die Radiusfraktur geheilt. Dagegen entwickelte sich im Bereich der Ulna eine hypertrophe Pseudarthrose (Abb. 4), die dann mittels Spongiosaplastik und Reosteosynthese mit einer 8-Loch-LC-DCP versorgt wurde (Abb. 5). Auch im weiteren Verlauf blieb die knöcherne Konsolidierung bei Lockerung und Bruch des Osteosynthesematerials aus (Abb. 6).

Zur Verbesserung der biomechanischen Stabilität führten wir nach komplizierter Entfernung der gelockerten und gebro-

OP-JOURNAL 2008; 24: 206-207

(c) Georg Thieme Verlag KG Stuttgart • New York DOI 10.1055/s-2008-1039126

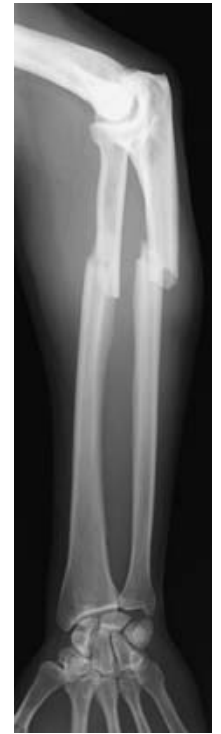

Abb. 1 Instabile Unterarmschaftfraktur im proximalen Drittel.

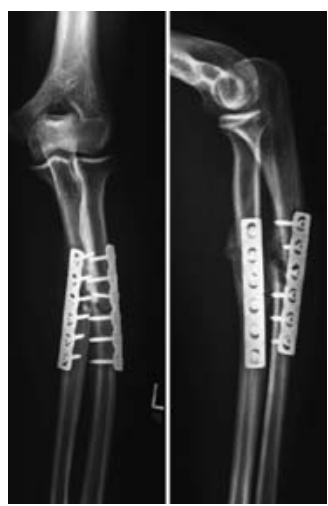

Abb. 3 Zwei Monate postoperativ zeigt sich eine hypertrophe Bruchheilungsstörung, die Ulna weist eine Angulationsfehlstellung auf.

chenen Schrauben (Abb. 7) einen Verfahrenswechsel mittels intramedullärer Nagelosteosynthese mit einem $5 \mathrm{~mm} \times$ $26 \mathrm{~cm}$ ForeSight ${ }^{\circledR}$-Nagel (Fa. Smith \& Nephew) durch (Abb.8). Die Achse der Ulna wurde korrigiert und der knöcherne Defekt mit autologer Spongiosa aufgefüllt. Im weiteren Verlauf zeigte sich im Bereich der Pseudarthrose nun eine vollständige knöcherne Konsolidierung (Abb.9), sodass der Nagel 13 Monate

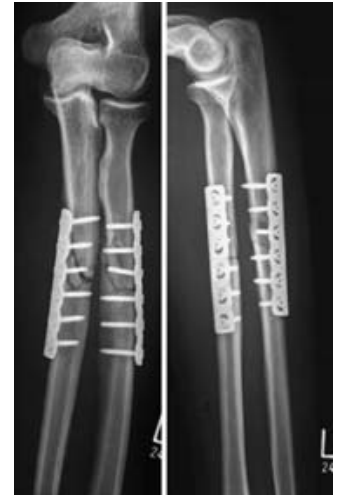

Abb. 2 Initiale Versorgung mittels LC-DCPlattenosteosynthese. Im Frakturbereich beider Knochen befinden sich mehrere knöcherne Fragmente. Die Stabilisierung beider Knochen ist im proximalen Bereich unzureichend, da faktisch jeweils nur 2 Schrauben die proximalen Fragmente fixieren.

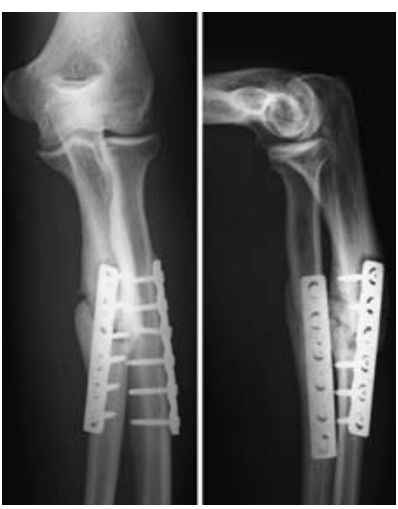

Abb. 4 Ein Jahr postoperativ zeigt sich eine knöcherne Konsolidierung des Radius. Im Bereich der Ulna hat sich eine hypertrophe Pseudarthrose entwickelt.

nach diesem Revisionseingriff entfernt werden konnte (Abb.10).

\section{Diskussion}

Aufgrund erheblicher Torsions- und Angulationskräfte gehören Plattenbrüche und Schraubenlockerungen zu den häufigsten Komplikationen nach operativer Versorgung von Unterarmfrakturen. Als wesentliche pathophysiologische Fak- 

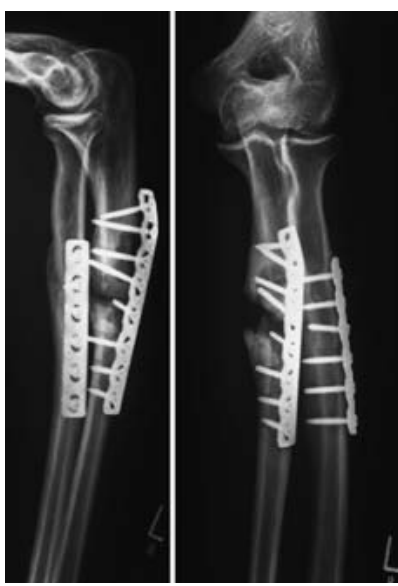

Abb. 5 Ergebnis der Reosteosynthese und Spongiosaplastik der Ulnapseudarthrose.

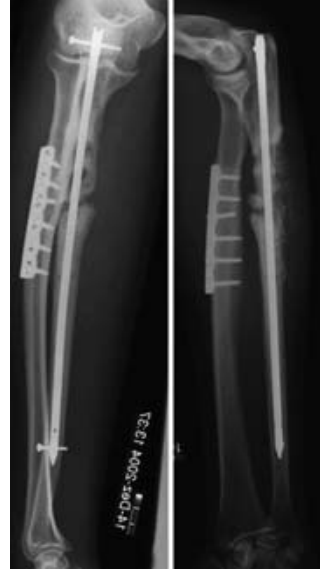

Abb. 8 Achsenkorrektur und Nagelosteosynthese der Ulna.

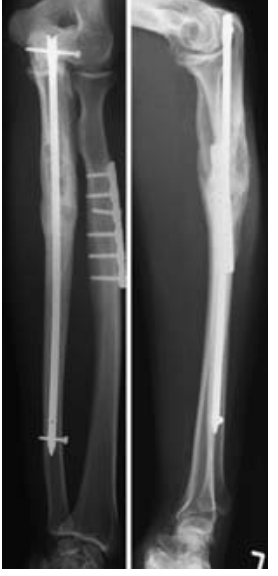

Abb. 9 Die Verbesserung der biomechanischen Stabilität ermöglicht die knöcherne Konsolidierung der Pseudarthrose.

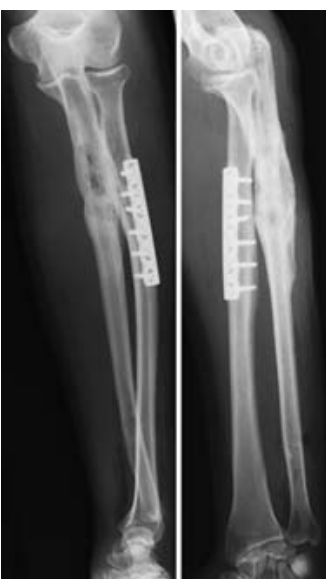

Abb. 10 Radiologisches Ergebnis nach Entfernung des Ulnanagels.

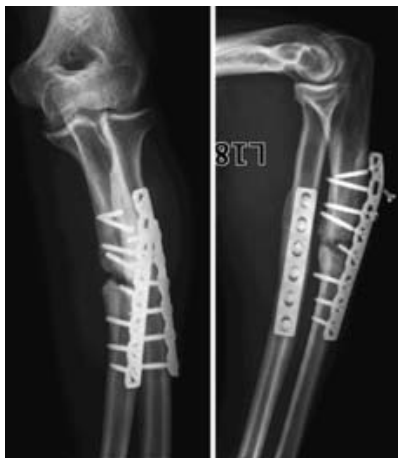

Abb. 6 Trotz deutlicher Kallusbildung heilt die Pseudarthrose im weiteren Verlauf nicht aus. Vielmehr zeigen sich hier Schraubenbrüche und Plattenlockerung.

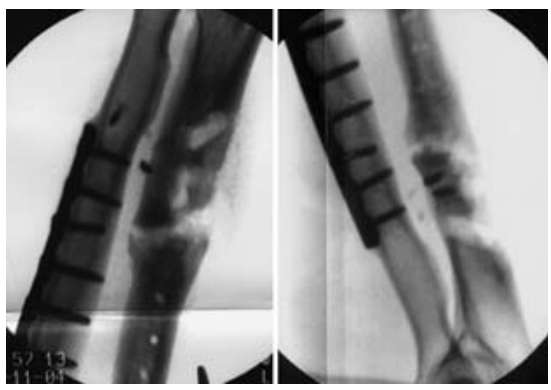

Abb. 7 Knöcherne Defekte als Folge der komplizierten Metallentfernung.

toren gelten neben der inadäquat durchgeführten Osteosynthese die Kortikalisatrophie und Porose, die durch die Störung der periostalen Durchblutung hervorgerufen werden. Abgesehen von der korrekten Wiederherstellung der Achsenverhältnisse und möglichst hoher Kompression des Frakturspalts muss bereits bei der Erstversorgung eine absolute Stabilität der Osteosynthese gewährleistet werden.
Zusätzliche Komplikationen sind im Rahmen der Revisionseingriffe zu erwarten (Abb. 7). Die Überbohrung und Entfernung der gelockerten und abgebrochenen Schrauben führen nicht nur zur Vergrößerung der Knochendefekte und Erhöhung der Instabilität, sondern auch zu einer zusätzlichen Irritation der Weichteile und der Durchblutungsverhältnisse. Die erneute operative Versorgung in solchen Situationen kann entweder durch eine stabilere Plattenosteosynthese oder durch eine intramedulläre Nagelosteosynthese erfolgen. Eine mögliche Indikation zur Nagelosteosynthese ergibt sich bei mehrfragmentären Frakturen, komplizierten Weichteilverhältnissen (Hautdefekte, Verbrennungen), manifesten Pseudarthrosen und Versagen der Plattenosteosynthese, Unterarmfrakturen bei mehrfach verletzten Patienten sowie bei osteopenen Knochenverhältnissen [1]. Aufgrund seiner Lage befindet sich das Implantat im biomechanischen Zentrum des Knochens und bietet als einen der wesentlichsten Vorteile beinahe die gleiche mechanische Stabilität in der Frontalund Sagittalebene bei einer langen Verankerungsstrecke innerhalb des Markkanals. Die Rotationsstabilität ist durch die statische bilaterale Verriegelung gegeben.

Trotz dieser Vorteile sind einige Probleme der Nagelosteosynthese der Unterarmfrakturen, wie die anspruchsvolle Operationstechnik und die komplikationsträchtige proximale Verriegelung am Radius, noch nicht gelöst. Hier besteht noch ein erheblicher Verbesserungsbedarf.
Fazit

Postoperative Komplikationen nach Plattenosteosynthesen der Unterarmfrakturen sind keine Rarität. Im Rahmen der Erstversorgung muss abgesehen von der korrekten Wiederherstellung der Achsenverhältnisse und hoher Kompression des Frakturspalts eine absolute Stabilität der Osteosynthese gewährleistet sein. Bei instabilen Verletzungen sollte eine Plattenosteosynthese über eine längere Strecke erwogen werden. Im Falle eines Osteosyntheseversagens und knöchernen Defekts sollte neben der Spongiosaplastik eine stabile Plattenosteosynthese durchgeführt werden. Die intramedulläre Nagelosteosynthese der Ulna kann bei mehrfragmentären Frakturen, komplizierten Weichteilverhältnissen oder Defektsituationen wesentliche Vorteile bringen.

\section{Literatur}

${ }^{1}$ Hofmann A, Hessmann MH, Rudig L, Küchle R, Rommens PM. Intramedulläre Nagelosteosynthese der Ulna in der Revisionschirurgie. Unfallchirurg 2004; 107: 583-593

\section{Dr. med. Alexander Hofmann Assistenzarzt}

Prof. Dr. med. Pol Maria Rommens Ärztlicher Direktor

Zentrum für Unfallchirurgie und Orthopädie Johannes-Gutenberg-Universität Mainz

Langenbeckstraße 1 55101 Mainz

E-Mail: hofmann.trauma-surgery@ gmx.net

E-Mail: rommens@ unfallklinik.uni-mainz.de 